\title{
Effects of screen filter on visibility of alphanumeric presentation on CRT and LCD monitors
}

\author{
Aleksandar Zunjic*, Ljiljana Ristic and Dragan D. Milanovic \\ University of Belgrade, Faculty of Mechanical Engineering, Kraljice Marije 16, 11000 Belgrade, Serbia
}

\begin{abstract}
In this paper, a research that deals with the problem of determining of the visibility of the alphanumeric presentation on CRT and LCD screens is presented, in conditions when the screen filters are used, and when they are not used. A methodology that can be used for determination of the visibility of alphanumeric symbols on VDT screens is presented. It is determined the existence of differences in visibility of alphanumeric presentations when screen filters are used, compared to conditions when they are not used. This difference was statistically significant, while the visibility of the presented alphanumeric symbols on CRT and LCD screens that use screen filters is lower than when they are not used. For this reason, the use of protective screen filter is recommended in circumstances where a very high level of glare exists, when the visual fatigue is common appearance and when a user performs a task that does not depend on the detection of stimuli whose RGB values are low.
\end{abstract}

Keywords: CRT monitor, LCD monitor, screen filter, visibility

\section{Introduction}

Addressing of issues of visibility of presented alphanumeric symbols can be called a first step in optimizing of the reception and processing of visual information, when working with video display terminals. In this regard, an information component of visibility, according to Sanders and McCormick [5] is associated with the amount of details on an object that a man can register during observation, as well as with the meaning of the observed object for a man as a result of the process of perception. For these reasons, in the area of human - computer interaction, it is necessary to design and use the screen and its additional equipment in a way that allows a clear seeing of presented information, with less strain and fatigue.

The basic purpose of the filters that are used for VDT screens is to reduce or eliminate the harmful effects of glare and reflection. Actually, the screen filters reduce the harmful effects of external light on the contrast and sharpness of the presentation on a screen. In addition, some protective screen filters have the function to reduce the level of radiation from the screen.

Visibility is an important characteristic that affects the possibility of detection of objects displayed on the VDT screen. Visibility can be descriptively determined as a visual quality of characters or symbols that make them different from their surroundings [5]. With regard to VDT screens, from the practical standpoint this relates to the possibility of alphanumeric character or symbol perception and distinction from its basis.

Recently, two types of anti-glare computer filters are used. These are: anti-reflection coated glass and anti-reflection coated plastic [2]. Given that the glare, to some extent, also exists on the LCD screens, VDT users most often positively declare themselves in relation to implementation of these screen filters. However, in the literature, the screen filter was primarily considered as the device that can reduce or eliminate certain detrimental effects on a VDT user. Nevertheless, it is difficult to find a research where

\footnotetext{
"Address for correspondence: Aleksandar Zunjic, Ph.D., Assoc. Prof., Department of Industrial Engineering, Faculty of Mechanical Engineering, University of Belgrade, Kraljice Marije 16, 11000 Belgrade, Serbia, Tel.: +381642537486; E-mail: azunjic@mas.bg.ac.rs
} 
it is considered whether and to what extent this factor influences the visibility, especially on LCD monitors. For that reason, this paper deals with the problem of determination of display visibility changes, due to the screen filter use. The main goal of this paper is to determine the existence of alphanumeric character visibility difference, with and without the screen filter on CRT and LCD monitors.

\subsection{Theoretical basis for applied research methodology}

For understanding of research methodology that is applied in this paper to study the visibility, first, from the theoretical aspect it is necessary to consider some terms and settings related to the color perception, trichromatic theory and RGB color space. These phenomena within this section are treated together because it is necessary to point out the relationship that exists between them.

Basic physical dimensions that describe the color of the light source are its wavelength, intensity and spectral purity [6]. Basic dimensions which from the point of view of perception correspond to the aforementioned physical dimensions are hue, brightness and the degree of saturation. Hue relates to the wavelength of light of certain colors based on which we can conclude that something is blue, green, etc. Hue corresponds to the usual experience of a color of an object or light [6]. The intensity of the light stimulus relates to the photometric quantity, and the sensation produced by such a stimulus is called brightness [7]. The degree of saturation is a relative amount (quantity) of hue, which contains a surface or an object [6]. This dimension refers to the purity of the color, i.e. to the level of deviation from the monochromatic light.

The relationship between the hue, brightness and saturation can be symbolically represented by model of color spindle [6]. On the vertical axis of the spindle, there are shades of gray, from black at the top of the cone that is facing down, until the completely white at the top of the cone that is facing upwards. This axis symbolizes brightness. Hue is represented over the circumference of a cone. The distance between the vertical axis and circumference of a cone (along the radius) symbolizes the degree of saturation, with less saturation of the hue at the greater distance from the vertical axis.

Trichromatic theory, whose founders are Young and Helmoltz, rests on the assumption that there are three types of receptors on the retina to detect colors, each of which is of different spectral sensitivity [6]. According to this theory, light of only three wavelengths is sufficient to produce a visual sensation of the entire visual spectrum. Helmholtz, who expanded and completed the initial Young's theory, held that there are three types of receptors, whose individual maximal sensitivities are achieved as a result of the appearance of blue, green and red color. This means that one type of receptor achieves maximum sensation on the occurrence of blue light, the other upon the occurrence of green and the third upon the occurrence of red light. All other sensations of colors appear as a result of reaction of only these three types of receptors, whereby they are activated in the appropriate proportion on the occurrence of any color in the visual spectrum. Strong support to this theory has given by the subsequent researches. It was found that the retina has the three types of cones, whose individual maximal sensitivity achieves on the occurrence of short wavelengths ( $\mathrm{S}$ - cones), medium ( $\mathrm{M}$ - cones) and long wavelengths ( $\mathrm{L}$ - cones). These receptors, such as Bullimore and colleagues have stated [1], sometimes otherwise are called blue, green and red cones, because they give the respond to the above-mentioned colors. Trichromatic theory is consistent with the fact that human being can perceive a white color, when the mentioned receptors are stimulated in equal proportion.

Blue, green and red colors are also called the primary colors of the visible spectrum. Additive system of mixing of lights of different colors is based on proportional combining of the primary colors of the visible spectrum, to produce any other color. VDT monitors use an additive system of mixing of the primary colors for the formation of any alphanumeric symbol or picture on the screen. In this sense, in the most common use is the so-called RGB color space, which is based on additive mixing of red, green and blue color. RGB color space is most commonly applied system for color determination, and it has been used in almost all software applications that generate the image. Similar to the trichromatic theory that is based on the existence of three types of receptors that are sensitive to light of red, green and blue colors, which are used for forming of an image on the cognitive level, VDT monitors use RGB system for forming image of an object on the screen, based on generating of only three abovementioned colors. Each pixel on a monitor screen is of dark color when it is not in use. However, when the red, green and blue components of pixels are electronically excited in a certain proportion, differ- 
ent colors of the visual spectrum are obtained. If the excitation of red, green and blue pigments performs in equal proportion and with the maximal intensity, we get the pure white light. In other words, by generating of wavelengths of red $(R)$, green $(G)$ and blue (B) color in a certain proportion, we get any color $(\mathrm{A})$, which can be expressed by equation $\mathrm{A}=$ $\delta \mathrm{R}+\varepsilon \mathrm{G}+\zeta \mathrm{B}[6]$. Coefficients of the proportion of the three wavelengths that belong to the primary colors of the visible spectrum are labeled with $\delta, \varepsilon$ and $\zeta$. In this way, different intensities of colors on the screen are generated by excitation of the red, green and blue pigment in different proportions.

Windows operating system enables specifying of a large number of colors based on changes in values for R, G and B, while each of these dimensions can take a value between 0 and 255 . In this way, colors and their intensities are defined through the corresponding RGB triplets. Each different combination of $R, G$ and $B$ values define another intensity of a certain color. In this way, it is possible to generate a $255 \times 255 \times 255$ color tones, or more than 16.5 million different colors. Pure black color has the RGB code $[0,0,0]$, while the pure white light with maximal intensity (brightness) has the code [255, 255, 255].

The method that was used for the study of visibility in this paper is based on determining of the absolute threshold for visual stimuli. For the purpose of research of the visibility of alphanumeric symbols, we have used the method of limit. The method of limit in accordance with the "stairs" experimental procedure consists of several steps. At the beginning, it is necessary to start from the value of the intensity of the stimulus that is below the expected value of the absolute threshold for that stimulus. The intensity of a stimulus then gradually increases in equal amounts until it reaches the value of the absolute threshold, i.e. until the moment when a subject detects the presence of stimulus. Detected value of the intensity of the stimulus then should be recorded. After the registration of the presence of the stimulus by the subject, starts the gradual decrease of the intensity of the stimulus in equal amounts, until the moment when the respondent cannot longer detect the presence of the stimulus. The value of the stimulus intensity at which the respondent cannot confirm the presence of stimulus is also a value of the absolute threshold, which should be recorded. After that, the intensity of the stimulus alternately increases and decreases in the series, with parallel recording of values of stimulus intensity at which the stimulus was detected, i.e. values at which the stimulus could no longer be detected. Value of the absolute thresh- old is then determined as the arithmetic mean of the values of absolute thresholds for each individual series.

\section{Method}

There is no single indicator or a physical dimension, which is used for the measurement and evaluation of the visibility. However, from the practical and theoretical viewpoint, as Sanders and McCormick quote [5], there is no difference between symbol visibility and detectability. This fact was used on the occasion of the research methodology selection. The essence of visibility determination consists in that to quantify the symbol discrimination from its surroundings, that is to say, the perception of alphanumeric character presented on the terminal screen in relation to its background or basis.

The software application is designed for that purpose, which represents a software adaptation of method of the limit, with application of the trichromatic theory, i.e. of RGB color space. The program is also designed to generate change in the $R, G$ and $B$ values, in equal proportions. Thus, the change was performed only along the vertical axis of the color spindle. In other words, the degree of brightness on the vertical axis of the color spindle was only changed, from totally black in the top of the cone that is facing down, up to the pure white color with maximal intensity in the top of the cone that is facing up. Between black and white light, program has enabled emission of 255 nuances of, conditionally gray color. In this way, intensity of the color was changed, i.e. the light emitted by monitors. As mentioned earlier, pure black color has the RGB code $[0,0,0]$, while the pure white light with maximal (brightness) has the code $[255,255,255]$. Any other color of an alphanumeric symbol (shade of gray) that has generated by the program has possessed its RGB code, in the form of triplets of values $[X, X$, $\mathrm{X}]$, where $\mathrm{X}>0$ and $\mathrm{X}<255$.

\subsection{Procedure of measurement}

Program for determining of the visibility has generated visual stimuli, in the form of alphanumeric symbols with $R, G$ and $B$ values, which were changed in the same way. In regard to the form of alphanumeric symbols, Verdana font was chosen, which in the literature states as a font suitable for VDT work. The font size of 10 pt was selected, 
which is in frequent practical application in performing of everyday tasks of the majority of users. Exposure time of a stimulus was $2 \mathrm{~s}$, which means that in a given time interval, an RGB value of the stimulus did not change. Step of change in stimulus intensity was one RGB unit. Alphanumeric characters are generated by a random function. Detection of a stimulus is enabled by the software, and it is realized by pressing the button $\mathrm{V}$, while the absence of a stimulus is registered by pressing the button $\mathrm{N}$. The background color on which alphanumeric character appeared was unchangeable and absolute black. At the beginning of the measurement, clearly visible alphanumeric character at the area around the middle of the screen was emitted, so that subjects knew where to direct a view in order to detect a symbol. Location of exposure of symbols did not change during the experiment.

Conditionally speaking, the experiment consisted of four parts. In the first part of the experiment (conditionally first), subjects were tested on the CRT screen without the screen filter. Instantly after activating the program, an alphanumeric character that was visible prior to the start of the experiment became invisible, because its RGB code now got zero value. In this way, the RGB value of an alphanumeric character is equalized with the RGB values of the background. Then, the RGB value of a new alphanumeric symbol (that is selected on a principle of a random selection) gradually began to increase for the unit value, with each new step (every two seconds). Due to the gradual increase in the stimulus intensity, the alphanumeric character gradually becomes visible. The task of subjects was relatively simple. At the moment, when a subject clearly and reliably was able to identify which alphanumeric character appeared on the screen, the task of a subject was to press the key V (which means "I see"), and at the same time to say out loud which alphanumeric character was seen (for example, "I see the letter A" ). The program has recorded automatically in table values of RGB triplets of displayed alphanumeric symbol, i.e. the value of the absolute threshold of visibility for this measurement. In addition, the program has recorded the generated alphanumeric character for the performed measurement. Immediately after the participant pressed the button $\mathrm{V}$, RGB value of the alphanumeric character was automatically increased for a certain amount above the absolute threshold, so that the symbol became very clear and visible. Immediately after that, the values of RGB triplet of the displayed character began to decline for the unit value in each subse- quent step. In this way, the visibility of alphanumeric characters gradually decreased, until the moment when the subject could not detect its presence on the screen. At this point, the task of subjects was to press the N key (which is meant, "I do not see"). The program has recorded automatically in table values of RGB triplets for which a subject was unable to register the presence of the alphanumeric character, i.e. the value of the absolute threshold for that measurement. After that, the program generated an alphanumeric symbol with zero RGB values, while the task of subjects was identical to the first measurement. This procedure was repeated ten times, which means that a total of ten values was measured for the absolute threshold of visibility. Values obtained for the absolute threshold were considered relevant, if all displayed alphanumeric symbols were successfully identified.

In the second part of the experiment (conditionally second), on the CRT monitor screen, which was used in the first part, the anti-reflection coated glass filter Aidata, model AG17AT was mounted. Experimental procedure in the second part of the experiment was identical to that in the first part of the experiment. In this way, per ten values for the absolute threshold of visibility was obtained for each subject, when the screen filter was used. In the third and fourth part of the experiment (conditionally third and fourth), identical procedure in relation to the previously described in the first two parts of the experiment was repeated for an LCD screen. For this part of the experiment, the anti-reflection coated plastic filter Targus Privacy Screen, model ASF170EU was used.

The sequence according to which the subjects performed two previous parts of the experiment was changed, to eliminate the possible occurrence of side effects. The experimental plan was formed under the principle of counter balancing. This means that every part of the previously described experiment is the same number of times performed at the first, second, third and fourth place.

In this research, 40 students of the University of Belgrade have participated. The subjects were between 22 and 45 years of age, with the average age of 27 years. All subjects had normal visual acuity. The experiment was performed in a room with artificial light sources. Illumination in the middle of the CRT and LCD screens was 210 lx. The reduction of direct component of glare was performed by placing curtains on the window surfaces and with other measures that were applicable. On the surfaces of the screens, there were no reflection and glare from 
natural or artificial light sources. The distance from subjects to the screens depended on their individual preferences (the distance of about $60 \mathrm{~cm}$ was suggested), but the distance for the same subject was not changed for all parts of the experiment. The time interval between measurements was about two minutes. A CRT monitor Samsung SyncMaster 793 of 17 ", with the resolution $1024 \times 768$, with the refresh rate of $60 \mathrm{~Hz}$ and a maximal luminance of 250 $\mathrm{cd} / \mathrm{m}^{2}$ was used, as well as an LCD monitor HP Compaq LE 1711 of 17", with the resolution $1024 \times 768$, with the refresh rate of $60 \mathrm{~Hz}$ and maximal luminance of $250 \mathrm{~cd} / \mathrm{m}^{2}$. All other cconditions of the experiment in all treatments were identical.

\section{Results}

The research results are presented in Figures 1 and 2. Figure 1 contains the results concerning the measurement of visibility of alphanumeric symbols that were displayed on the CRT screen with the screen filter (light-colored columns), and without the screen filter (darker colored columns). Figure 2 contains the results concerning the measurement of visibility of alphanumeric symbols that were displayed on the LCD screen with the screen filter (light-colored columns) and without the screen filter (darker colored columns). Numerical values for the absolute threshold of visibility are in RGB units and represent the average values per subject for 10 measurements. Due to the fact that in the experiment were performed the change of the intensity of visual stimulus through changing the degree of brightness along the vertical axis of the color spindle, values of RGB triplets are identical. In other words, it means that the values of individual measurements for $R, G$ and $\mathrm{B}$ in determining of the threshold of visibility were the same. Due to the compact presentation, the picture shows only one numerical value for each RGB triplet.

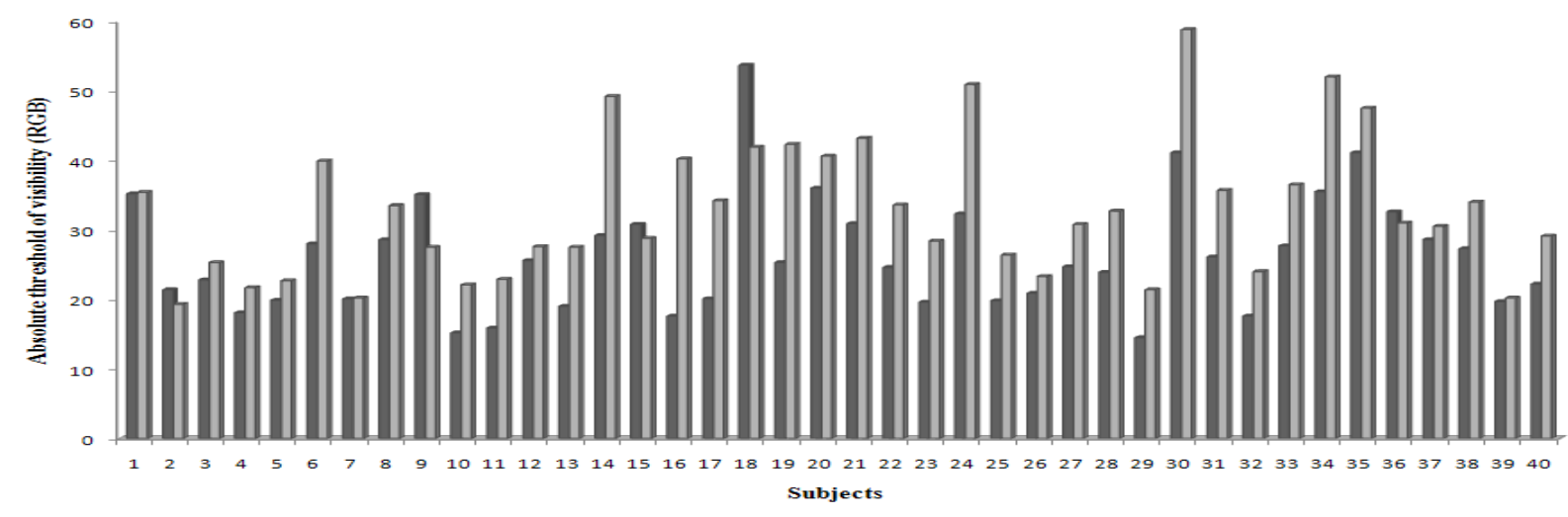

Fig.1. The absolute threshold of visibility of alphanumeric symbols that were presented on the CRT screen of a VDT with and without the screen filter.



Fig. 2. The absolute threshold of visibility of alphanumeric symbols that were presented on the LCD screen of a VDT with and without the screen filter. 
Table 1 shows the mean values for the threshold of visibility for the CRT screen without screen filter, for $\mathrm{CRT}_{\text {wf }}$ screen with screen filter, for LCD screen without screen filter and for $\mathrm{LCD}_{\mathrm{wf}}$ screen with screen filter. Mean values for the threshold of visibility were obtained from the measurement results for all 40 subjects.

Table1

Mean values for the threshold of visibility for all four mediums on which the measurements were performed.

\begin{tabular}{lccc}
\hline \multicolumn{4}{l}{ Mean of visibility threshold (RGB) } \\
\hline CRT & CRT $_{\text {wf }}$ & LCD & LCD $_{\text {wf }}$ \\
26.21 & 32.82 & 28.05 & 31.72 \\
\hline
\end{tabular}

\subsection{Analysis}

In order to determine the existence of the visibility difference regarding the alphanumeric characters, which are presented on the VDT screens with and without the filter, it is necessary to compare the absolute thresholds of visibility. If the visibility threshold is higher, the visibility of the alphanumeric character is lower and vice versa. For both VDT monitors the null hypothesis is formed that the absolute threshold of visibility with screen filters is equal or less than the threshold of visibility without screen filters.

We have used the t-test for dependent samples with paired observations in both cases. Bearing in mind that the experiment was repeated with the same subjects, the samples are dependent [3]. Random variables between individual pairs are independent values in this case. The paired observations are applied in order to eliminate the influence of the characteristics of subjects to the results of measurements. However, before the application of paired two-sample t-test, it is necessary to verify computationally whether are also satisfied other conditions for its implementation. In this regard, it is necessary to verify the condition for the application of this test, which refers to the necessity that sampling distribution of the $d_{i}$ possesses a normal distribution [4]. In this case, $d_{i}$ is the difference between the measured values for the threshold of visibility for the screens with filter and the screens without filter.

In order to check the normality of distribution for $\mathrm{d}_{\mathrm{i}}$, we will apply the Shapiro-Wilk normality test. First, we will test the normality of distribution for $d_{i}$ in the case of using the CRT monitor with the screen filter and the CRT monitor without the screen filter. The $\mathrm{W}$-test statistic in this case equals 0.975 . For the level of significance of 0.05 , tabular value [8] of the $\mathrm{W}$-test equals 0.940 . Because the calculated value for $\mathrm{W}$ is greater than the tabular, the null hypothesis according to which values for $\mathrm{d}_{\mathrm{i}}$ posses a normal distribution is accepted.

Now we will test the normality of distribution for $\mathrm{d}_{\mathrm{i}}$ in the case of using the LCD screen with the screen filter and the LCD screen without the screen filter. The $\mathrm{W}$-test statistic in this case equals 0.941 . For the level of significance of 0.05 , tabular value of the $\mathrm{W}$ test equals 0.940 . Because the calculated value for $\mathrm{W}$ is greater than the tabular, the null hypothesis according to which values for $d_{i}$ posses a normal distribution is accepted.

Taking into account that all the requirements for the application of paired two-sample t-test are satisfied, firstly, we will determine whether exists a statistically significant difference in visibility between the CRT screen with the screen filter and the CRT screen without the screen filter. The value for $t$ obtained by calculation in this case was 5.775. For the level of significance of 0.05 , tabular value of t-test equals 1.68. Since the calculated value for $t$ is greater than the tabular, the null hypothesis is rejected, and the alternative hypothesis is accepted, according to which the absolute threshold of visibility of alphanumeric symbols when it is in use the CRT screen with the screen filter is greater than the absolute threshold of visibility of alphanumeric symbols when it is in use the CRT screen without the screen filter. This means that the visibility of the displayed alphanumeric characters on the CRT screen with the screen filter is less than the visibility of alphanumeric characters displayed on the CRT screen without the screen filter.

Now we will test whether there is a statistically significant difference in visibility between the LCD screen with screen filter and the LCD screen without the screen filter. The value for $t$ obtained by calculation in this case was 3.67. For the level of significance of 0.05 , tabular value of t-test equals 1.68. Since the calculated value for $t$ is greater than the tabular, the null hypothesis is rejected, and the alternative hypothesis is accepted, according to which the absolute threshold of visibility of alphanumeric symbols when it is in use the LCD screen with the screen filter is greater than the absolute threshold of visibility of alphanumeric symbols when it is in use the LCD screen without the 
screen filter. This means that the visibility of the displayed alphanumeric characters on the LCD screen with the screen filter is less than the visibility of alphanumeric characters displayed on the LCD screen without the screen filter.

\section{Discussion}

Taking into account the average values (for all 40 subjects) for the threshold of visibility of alphanumeric symbols, the impairment of visibility in the case of CRT monitors with screen filter of $20.14 \%$ is obtained, while in the case of LCD monitors with screen filter, it has the value of $11.57 \%$. Although VDT monitors and their features often were the subject of ergonomic researches, a study that have dealt with the problem of determining of the visibility of alphanumeric symbols is not known, when the screen filters are in use on CRT and LCD monitors. Studies that have dealt with testing of various characteristics of screen filters are also known. However, these studies also did not take into account the visibility factor. For this reason, the results obtained in this study cannot be compared with some previous results obtained in research of this kind.

\section{Conclusion}

In this paper, a method that can be used for determination of the visibility of displayed alphanumeric symbols on VDT screens is presented. Given the results, we can conclude that the visibility of CRT and LCD screens are smaller when using anti- reflection filter. This difference was statistically significant. Given this, the use of protective screen filter is recommended in circumstances where a very high level of glare exists, when the visual fatigue is common appearance and when a user performs a task that does not depend on the detection of stimuli whose RGB values are low.

\section{Acknowledgements}

We wish to express our appreciation to the participants of this research. This work is supported in a part by a grant from the Ministry of Science and Technological Development, Serbia.

\section{References}

[1] M.A. Bullimore, P.A. Howarth and E. J. Fulton, Assessment of visual performace, in: Evaluation of human work - a pracical ergonomics methodology, J. R. Wilson and E. N. Corlett, ed., Taylor \& Francis, London, pp. 804-839.

[2] S. Middendorf, Addendum: A case for anti-glare computer filters, in: Visual ergonomics handbook, J. Anshel, ed., CRC Press, Boca Raton, 2005, pp. 53-62.

[3] R. Njegic, M. Lovric, M. Zizic and D. Pavlicic, Fundamentals of statistical analysis (in Serbian), Savremena administracija, Belgrade, 1991

[4] R.L. Ott and M. Longnecker, An introduction to statistical methods and data analysis, Thomson Learning, Inc., Pacific Grove, CA, 2001.

[5] M.S. Sanders and E.J. McCormick, Human factors in engineering and design, McGraw-Hill, New York, NY, 1993.

[6] H.R. Schiffman, Sensation and perception, John Wiley \& Sons, Inc., New York, 1996.

[7] D. Schreuder, Outdoor lighting: physics, vision and perception, Springer, Heidelberg, 2008.

[8] S.S. Shapiro and M.B. Wilk, An analysis of variance test for normality, Biometrika 52 (1965), 591-611. 